\title{
Deep blue light emission of (4,3-Oxo-HCMM) coumarin derivative for organic LED display application
}

Cite as: AIP Conference Proceedings 2100, 020105 (2019); https://doi.org/10.1063/1.5098659

Published Online: 25 April 2019

A. G. Pramod, Y. F. Nadaf, C. G. Renuka, and M. Sucheta

\section{ARTICLES YOU MAY BE INTERESTED IN}

Committee: Prof. Dinesh Varshney memorial National Conference on Physics and Chemistry of Materials (NCPCM2018)

AIP Conference Proceedings 2100, 010002 (2019); https://doi.org/10.1063/1.5098554

OLED display application of 2-(6-Fluoro-2-oxo-2H-chromen-4-ylmethoxy)-benzoic acid methyl ester derivative

AIP Conference Proceedings 2100, 020106 (2019); https://doi.org/10.1063/1.5098660

$\mathrm{TiO}_{2}$ microstructures synthesized via solgel process for dye-sensitized solar cell applications AIP Conference Proceedings 2100, 020104 (2019); https://doi.org/10.1063/1.5098658

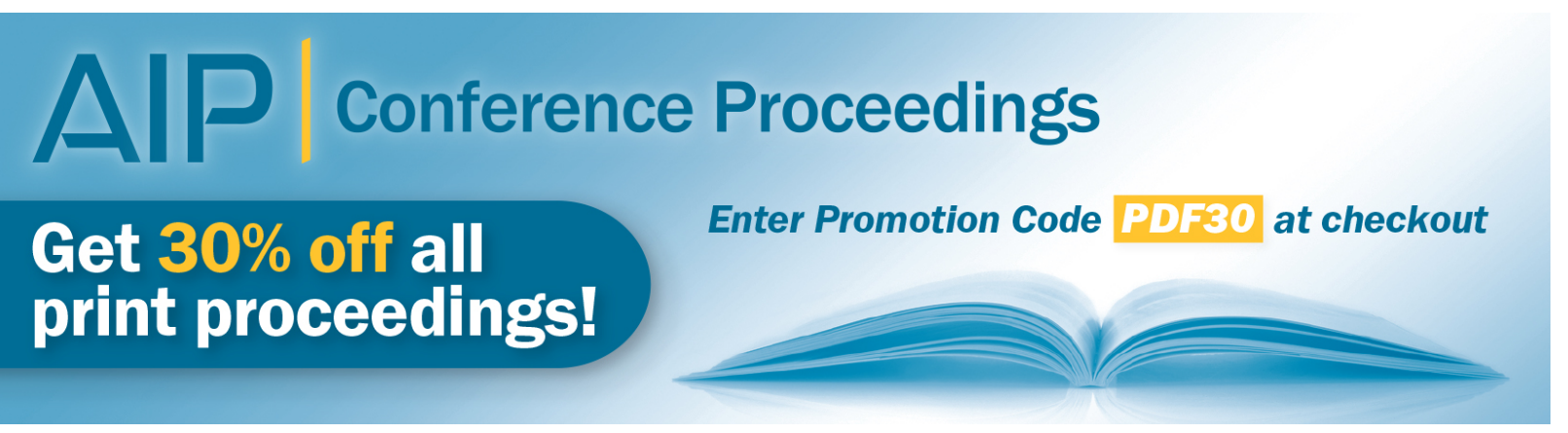




\title{
Deep Blue Light Emission of (4,3-Oxo-HCMM) Coumarin Derivative for Organic LED Display Application
}

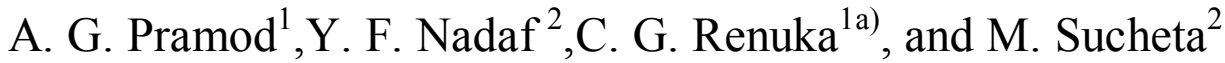 \\ ${ }^{1}$ Department of Physics, Jnanabharathi Campus Bangalore University, Bengaluru-560056 India \\ ${ }^{2}$ Department of Physics and Research Center, Maharani Science College for Women, Bengaluru-560001India \\ a) Corresponding author: renubub@gmail.com
}

\begin{abstract}
In the present technological world huge demand of white light emitting diodes has received much importance due to their vast applications in various sensors, lightning devices and display etc. The white light can be produced by mixing exact amount of green, blue and red light. Therefore, we made an attempt to produce a LED application making use of organic compounds. Hence, we synthesized 4-Hydroxy-3-[(4-Hydroxy-2-Oxo-2H-Chromen-3-yl) (4Methoxyphenyl) Methyl]-2H-Chromen-2-One (4,3-Oxo-HCMM). In this present work we report, the 4,3-Oxo-HCMM possesses high color purity, good CIE chromaticity coordinate, and they would have potential organic light emitting devices (LED) application, this simple method to produce the blue light as blue component can play important role in WLED. Uv-Vis absorption spectra is used to determine the optical energy bandgap Eg, andfrom the photoluminescence spectra duality nature of wavelengths for4,3-Oxo-HCMM molecule in different solvents is obtained due to solvation effect, this result shows a simple extraction of dye in different solvents which can be used to produce the desired wavelength.
\end{abstract}

\section{INTRODUCTION}

A great effort was put by many researchers in synthesis and design of new organic semiconductors (OS), while trying to find the synthesis process of low cost electronic compositions. A vast organic molecules was tested in this concern, including some p-extended coumarins. The effect of solvents should be understood in detail intending to understand and estimate the performance of electronic devices in different media. Hence, the Semiconductors (SCs) have interesting and significant optoelectronic properties for various technologies such as detectors, transistors, microelectronics, lasers ${ }^{1-3}$, photovoltaics and diodes. Organic semiconductors (OSCs) have been widely used in energy conversion and medicine. For this, among various derivatives of coumarins, bis-coumarins have drawn much importance since they are well known to possess distinct photophysics, pharmacological and biological properties viz., antifungal, anti-HIV, antibiotic, antiviral, antibacterial, anticoagulant, anticlotting and anticancer and also proved several applications in different fields. Further, the biscoumarin derivatives has fascinated the scientific community due to the interesting applications of synthesized compounds (bis) such as blue and green OLEDs have met the requirements for practical applications ${ }^{2}$. Therefore, we attempted to synthesis4-Hydroxy-3-[(4-Hydroxy-2Oxo-2H-Chromen-3-yl) (4-Methoxyphenyl) Methyl]-2H-Chromen-2-One (4,3-Oxo-HCMM) dye using acetic acid as a catalyst. Furthermore, the electronic spectra of 4,3-Oxo-HCMM derivative in solutions depending on the solvents parameters have showed excellent fluorescence properties. These derivatives also exhibit the phenomena such as inter molecular charge transfer (ICT) and excited state intermolecular proton transfer which were played a major role in view of excellent photophysical properties like large stoke shifts, significant photostability and intense luminescence ${ }^{4}$. However, in literature there is no considerable report on the fluorescence studies of 4,3-Oxo-HCMM bis coumarin compound. To this end, the present paper reports on the optical, fluorescence properties, Commission Internationale de I'Eclairage (CIE) and color purity of previously mentioned compound dissolved in different solvents. 


\section{EXPERIMENTAL}

\section{Materials and Methods}

All the solvents used in this present study (spectroscopic grade) such as 1,4 Dioxane, Hexadecane, Acetonitrile, Butyl acetate, Butanol and Octanol were purchased from the local chemical supplier having $99.9 \%$ purity were used without purification. The UV-Vis absorption spectra was collected from UV-1800 (Shimadzu) spectrophotometer and steady-state fluorescence experiment was carried out making use of Hitachi F-2700 Fluorescence Spectrofluorophotometer. The selected excitation wavelength is used for recording the fluorescence emission and obtained more resolution at the highest intensity. The experiment (all measurements) were carried out at 274 Kelvin with solution concentration of $10^{-6}-10^{-7} \mathrm{M}$ to overcome inner filter effect and self-aggregation effect.

\section{Synthesis Procedure}

The titledbiscoumarin derivative was synthesized by catalyst method choosing the acetic acid as a catalyst. The mixture of 4-hydroxycoumarin $(4 \mathrm{mmol})$, aryl aldehydes $(2 \mathrm{mmol})$ and Acetic acid $(10 \mathrm{~mL})$ was taken in a $200 \mathrm{~mL}$ beaker and refluxed for 2-3 hrs. By using thin layer chromatography (TLC) (Eluent: n-hexane and Ethyl acetate) the progress of the reaction was monitored. The vanishing of solvent in vacuum afforded the crude product which was recrystallized from ethanol to get 4-Hydroxy-3-[(4-Hydroxy-2-Oxo-2H-Chromen-3-yl) (4-Methoxyphenyl) Methyl]$2 \mathrm{H}-\mathrm{Chromen}-2-\mathrm{One}$ biscoumarins. The optimized structure of synthesized4,3-Oxo-HCMM is presented in Fig. 1.

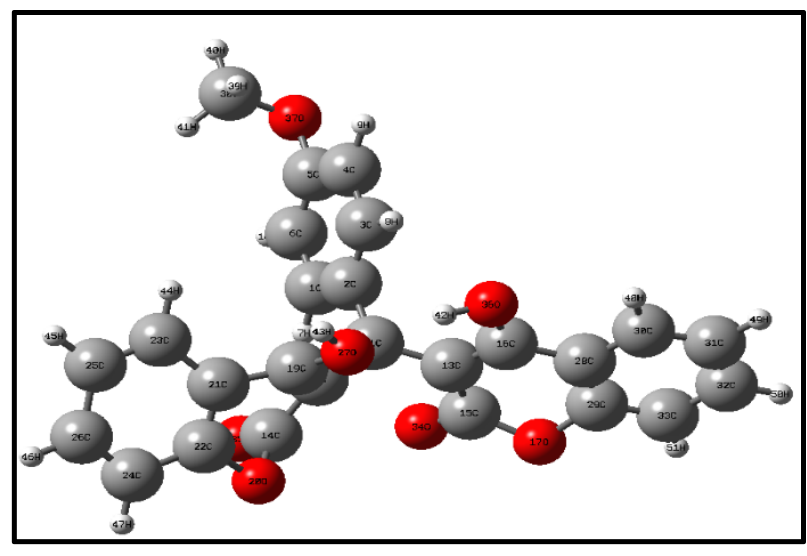

FIGURE 1.Optimized Structure of 4,3-Oxo-HCMM Derivative.

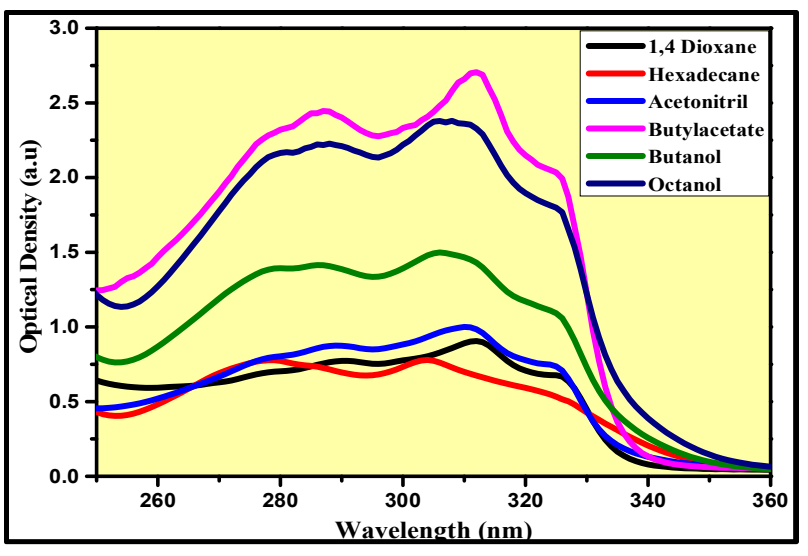

FIGURE 2.UV-Vis Absorption spectra of 4,3-Oxo-HCMM Derivative.

\section{RESULTS AND DISCUSSION}

The UV-Vis absorption spectra (Fig. 2.) was recorded and the optical properties of titled derivative was studied and from these spectra we estimated the bandgap energy of the studied derivative so that, the plots of $(\alpha h v)^{2}$ versus photon energyhv for solution phase of titled compound is depicted in Fig. 2. The linearity observed from the graph for all studied solvents confirms that electronic transitions are direct allowed transitions. Hence, the linearity is shown by the arrows. Energy band gap Eg is determined by means of extrapolating the linear region ${ }^{3}$ of Fig. 3 . The measured Eg values are presented in Table 1. And photoluminescence (PL) emission spectra of the synthesized 4,3Oxo-HCMM coumarin derivative in different organic solvents of varying dielectric constant and refractive index such as 1,4 dioxane, hexadecane, acetonitrile, butyl acetate, butanol and octanol.The PL spectra of synthesized4,3Oxo-HCMM presented in Fig. 4. The sample was excited at $320 \mathrm{~nm}$ wavelength and the corresponding PL spectra shows the dual emission peaks observed in all the studied solvents. In case of 1,4 Dioxane and Hexadecane solvents, the first peak is obtained at around $403 \mathrm{~nm}$ and the second peak which is highly intense and sharp peak at $429 \mathrm{~nm}$. In case of Acetonitrile the emission peak is obtained at $455 \mathrm{~nm}$. Whereas, in case of Butanol and Octanol the first emission peak is found at $346 \mathrm{~nm}$ and the second peak is found at $455 \mathrm{~nm}$. Also from Fig 3, it is clear that the emission spectra showed a slight bathochromic shift (red shift) in all studied solvents, these shifts are probably due 
to increase in the polarity of the solvents indicates $\pi \rightarrow \pi^{*}$ transitions are involved and also suggesting that the involvement of photo induced intermolecular charge transfer (ICT) state, apart from solvent polarity, the hydrogen bonding effect also plays a major role for the shift of emission bands. And also the observed phenomenon i.e., duality nature of wavelengths in 4,3-Oxo-HCMM molecule in different solvents is due to solvation effect, this results shows a simple extraction of dye in different solvents can be used to produce the desired wavelength ${ }^{4}$.

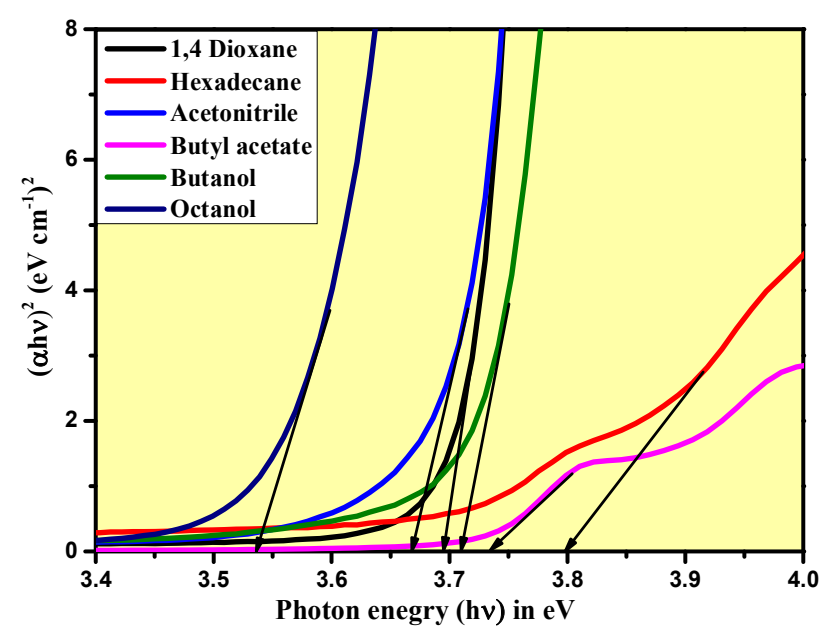

FIGURE3.Typical plot of $(\alpha h v)^{2}$ versus Photon energy for direct band gap measurements.

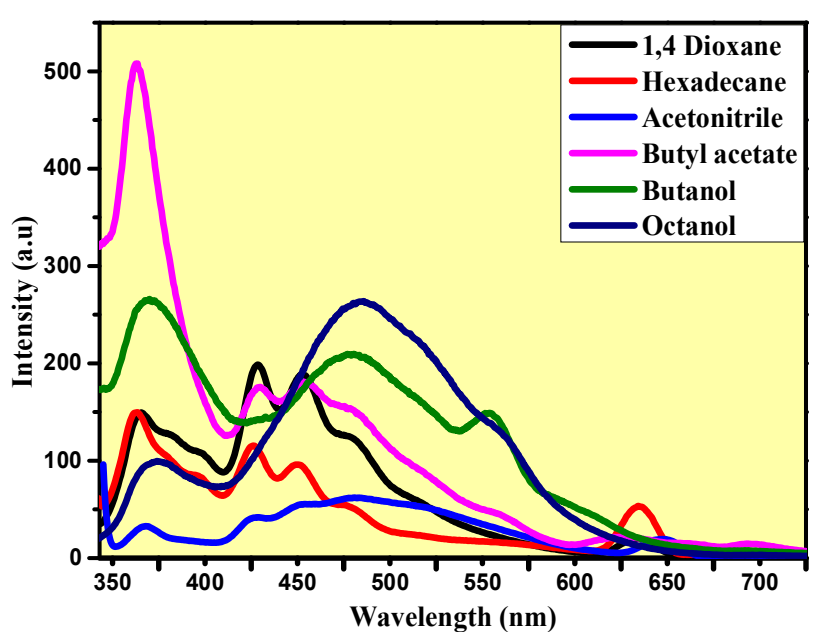

FIGURE4. The photoluminescence emission spectra 4,3Oxo-HCMM.

In order to determine the color coordinate and color purity of 4,3-Oxo-HCMM derivative, The Commission Internationale de I'Eclairage (CIE) color coordinate values has been determined by using Osram Sylvania CIE coordinates software 5 . It is clear from the Fig. 5 that the CIE coordinates lies in deep blue region for 1,4 Dioxane, Hexadecane, Acetonitrile, Butyl acetate, Butanol and Octanol the corresponding $\mathrm{x}$ and $\mathrm{y}$ values are tabulated in Table 1. The color purity is an important property to estimate the possibility of the newly synthesized 4,3Oxo-HCMM derivative for OLEDs and display applications ${ }^{6}$. The color purity of the 4,3-Oxo-HCMM is calculated using the following equation used below.

\begin{tabular}{lcccccc}
\hline Solvents & $\begin{array}{c}\lambda_{\mathrm{a}} \\
(\mathbf{n m})\end{array}$ & $\begin{array}{c}\boldsymbol{\lambda}_{\mathrm{e}} \\
(\mathbf{n m})\end{array}$ & $\begin{array}{c}\text { Band gap } \\
\mathbf{E}_{\mathbf{g}}(\mathbf{e V})\end{array}$ & \multicolumn{2}{c}{ CIE Coordinates } & \multirow{2}{c}{$\begin{array}{c}\text { Color } \\
\text { purity }(\%)\end{array}$} \\
\cline { 5 - 6 } & & & & $\mathbf{x}$ & $\mathbf{y}$ & \\
\hline 1,4 Dioxane & 312 & 404 & 3.69 & 0.1778 & 0.1127 & 80.83 \\
Hexadecane & 280 & 339 & 3.80 & 0.2638 & 0.1808 & 30.34 \\
Acetonitrile & 310 & 455 & 3.66 & 0.1977 & 0.2237 & 57.25 \\
Butyl acetate & 310 & 388 & 3.73 & 0.1722 & 0.1257 & 79.05 \\
Butanol & 306 & 458 & 3.71 & 0.1761 & 0.2089 & 67.99 \\
Octanol & 308 & 458 & 3.53 & 0.1717 & 0.2219 & 66.11 \\
\hline
\end{tabular}

Table 1. CIE color coordinates with color purity of 4,3-Oxo-HCMM in studied solvents.

Where, $(\mathrm{X}, \mathrm{Y})$ are CIE chromaticity coordinate values of studied compound, $\left(\mathrm{X}_{\mathrm{a}}, \mathrm{Y}_{\mathrm{a}}\right)$ is the color coordinate of white illumination and $\left(\mathrm{X}_{\mathrm{b}}, \mathrm{Y}_{\mathrm{b}}\right)$ is the CIE coordinate of the dominant emission wavelength. In this report the $\left(\mathrm{X}_{\mathrm{a}}, \mathrm{Y}_{\mathrm{a}}\right)=$ $(0.310,0.316),\left(\mathrm{X}_{\mathrm{b}}, \mathrm{X}_{\mathrm{b}}\right)=(0.14,0.08)$, color purity and the $(\mathrm{X}, \mathrm{Y})$ values are presented in Table 1 . From Table 1 it shows the color purity $\%$ at excitation wavelength of $320 \mathrm{~nm}$ for studied solvents.

$$
\text { Color purity }=\frac{\sqrt{\left(\mathrm{X}-\mathrm{X}_{\mathrm{a}}\right)^{2}+\left(\mathrm{Y}-\mathrm{Y}_{\mathrm{a}}\right)^{2}}}{\sqrt{\left(\mathrm{X}_{\mathrm{b}}-\mathrm{X}_{\mathrm{a}}\right)^{2}+\left(\mathrm{Y}_{\mathrm{b}}-\mathrm{Y}_{\mathrm{a}}\right)^{2}}} \times 100 \%
$$

However, this results revealed that the observed the color coordinates of 4,3-Oxo-HCMM slightly vary with different solvents due to decrease in intensity as well as the area of the photoluminescence emission. Thus, the 4,3Oxo-HCMM possesses high color purity and good CIE chromaticity coordinate also they would have potential 
application organic light emitting devices, this simple method to produce the blue light as blue component can play important role. The calculated chromaticity coordinates for 4,3-Oxo-HCMM in chloroform are near to standard chromaticity (NTSC) for excellent blue color and thus are promising blue producers for WLED application ${ }^{7}$.
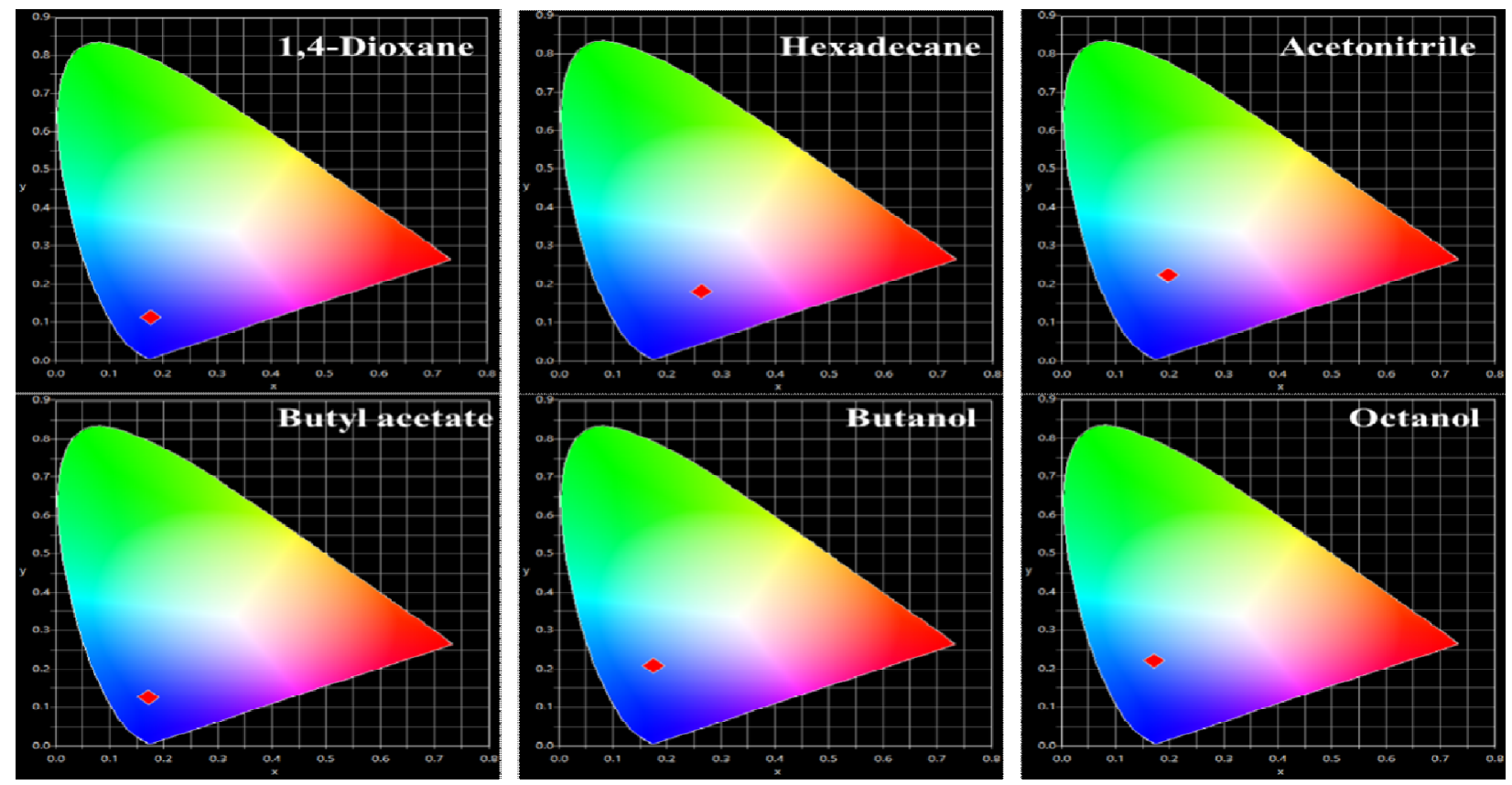

FIGURE 3. CIE Plots of 4,3-Oxo-HCMM in studied solvents.

\section{CONCLUSION}

The titled derivative 4-Hydroxy-3-[(4-Hydroxy-2-Oxo-2H-Chromen-3-yl) (4-Methoxyphenyl) Methyl]-2HChromen-2-One has been synthesized. Experimental bandgap energy $\mathrm{E}_{\mathrm{g}} \mathrm{of}$ the titled derivative was calculated using UV-Vis absorption spectra, from this we may conclude that the $E_{\mathrm{g}}$ is one of the important parameter for fabricating optoelectronic devices. The photoluminescence emission spectra showed a slight bathochromic shift (red shift) in the studied solvents, these red shifts are probably due to increase in the polarity of the solvents and suggesting that the involvement of photo induced intermolecular charge transfer (ICT) state, andthose duality nature of wavelengths in 4,3-Oxo-HCMM molecule in different solvents is due to solvation effect, this results shows a simple extraction of dye in different solvents can be used to produce the desired wavelength. The 4,3-Oxo-HCMM possesses high color purity, good CIE chromaticity coordinate and they would have potential application in organic light emitting devices, this simple method is used to produce the blue light as blue component and can play important role. The calculated chromaticity coordinates for 4,3-Oxo-HCMM in 1,4-Dioxane are near to standard chromaticity for excellent blue color and thus are promising blue producers for WLED application.

\section{REFERENCES}

1. A.R. Kiasat and L. Hemat-Alian, Res. Chem. Intermed. 41, 873 (2015).

2. J.R. Yerrabelly, V. Chakravarthula, H. Yerrabelly, P.R. Chitneni, V.R. Batchu, J.B. Nanubolu, and R.K. Krishnan, Tetrahedron Lett. 56, 2180 (2015).

3. C.G. Renuka, K. Shivashankar, P. Boregowda, S.S. Bellad, M. V Muregendrappa, and Y.F. Nadaf, J. Solution Chem. 46, 1535 (2017).

4. A.G. Al-Sehemi, M. Pannipara, and A. Kalam, Spectrochim. Acta Part A Mol. Bioml. Spectrosc. 171, 97 (2017).

5. $\quad$ C.G. Renuka, Y.F. Nadaf, G. Sriprakash, and S.Rajendra Prasad, J. Fluoresc. 28(3) 839 (2018).

6. See Osram Sylvania for CIE co-ordinates software, (2017), available at: https://www.sylvania.com.

7. Xiaoyong Huang, HengGuo, Bin Li J. Alloys Compd. 720, 29 (2017). 